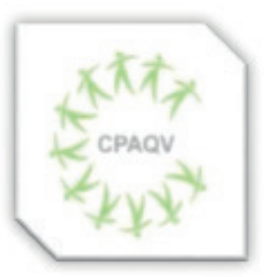

ISSN: $2178-7514$

\title{
A HEMORRAGIA SUBARACNÓIDE E AS CONDUTAS DO ENFERMEIRO DE ALTA COMPLEXIDADE
}

\section{Subarachnoid hemorrhage and high complexity nurse conduct}

\author{
Mayckow Carvalho da Silva Oliveira ${ }^{1}$; Bruno da Silva Lourenço ${ }^{2}$; \\ Janaina Rosa Lourenço da Silva ${ }^{3}$; Elizeu Adriano Rocha da Silva ${ }^{4}$; Anderson Costa Botelho ${ }^{5}$; \\ Márcia Cristina Marques Pereira da Silva ${ }^{6}$; Juliana Gomes dos Santos ${ }^{7}$
}

Vol. 13| No. 2| Ano 2021

\section{RESUMO}

Objetivos: Identificar os elementos que caracterizam a HSA e propor medidas de atendimento às vítimas com suspeita de HSA na sala de Emergência. Metodologia: Estudo exploratório e revisão bibliográfica. Os dados foram obtidos através de pesquisas em meio virtual na Biblioteca Virtual da Saúde, utilizando mais especificamente as base de dados da SCIELO, BDENF e MED LINE onde foram analisados e selecionados os trabalhos relacionados a este assunto. Resultados: A hemorragia subaracnóide (HSA) aneurismática é gerada principalmente pela ruptura de aneurismas saculares, os quais se referem às lesões usualmente adquiridas, que resultam do estresse hemodinâmico prolongado e concomitante degeneração arterial nos pontos de saída dos principais ramos arteriais, bem como nas bifurcações das maiores artérias cerebrais que cursam nas cisternas e nos espaços subaracnóideos. Destaca-se que dentre as complicações decorrentes das hemorragias subaracnoides cerebrais, a mais prevalente é o vasoespasmo. Conclusão: $\mathrm{O}$ enfermeiro é fundamental para conduzir a equipe de enfermagem no atendimento a pacientes com quadro de HSA, e considera-se que sua tomada de decisão deve ser rápida, o atendimento ao paciente deve ser sincronizado, exigindo do mesmo conhecimento científico e competência técnica para lidar melhor com o quadro.

Palavras-chave: Hemorragia cerebral, Traumatismos encefálicos, Cuidado de Enfermagem.

\begin{abstract}
Objectives: To identify the elements that characterize SAH and measures to assist victims with suspected SAH in the emergency room. Methodology: Exploratory study and literature review. Data were obtained through searches in a virtual environment in the Virtual Health Library, using more specifically as a database of SCIELO, BDENF and MED LINE where works related to this subject were locked and selected. Results: Aneurysmal subarachnoid hemorrhage (SAH) is mainly generated by the rupture of saccular aneurysms, which are characterized by the usually acquired characteristics, which result from prolonged hemodynamic stress and concomitant arterial degeneration at the exit points of the main arterial branches, as well as at the bifurcations of the largest cerebral arteries that course in the cisterns and subarachnoid spaces. It is noteworthy that among the complications resulting from cerebral subarachnoid hemorrhage, the most prevalent is vasospasm. Conclusion: The nurse is essential to lead a nursing team in the care of patients with SAH, and it is considered that their decision-making must be quick, patient care must be synchronized, requiring the same scientific knowledge and technical competence to better handle the frame.
\end{abstract}

Keywords: Brain hemorrhage, Brain injuries, Nursing care.

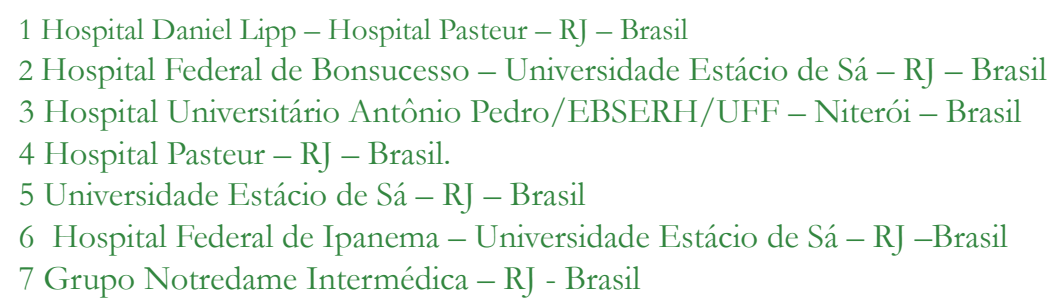

Autor de correspondência

Bráulio Nascimento Lima - brauliolima@outlook.com

DOI: $10.36692 / \mathrm{v} 13 \mathrm{n} 2-14 \mathrm{R}$ 


\section{INTRODUÇÃO}

Observa-se que a causa mais comum de hemorragia no espaço subaracnóideo se refere ao traumatismo cranioencefálico (TCE), sendo que a hemorragia subaracnóidea espontânea (HSA) é referente ao sangramento que acontece no espaço subaracnóideo decorrente de qualquer etiologia que não seja o traumatismo cranioencefálico ${ }^{1,2}$.

Cabe destacar que a principal causa de HSA é a ruptura de aneurismas intracranianos. É diretamente responsável por mais de 50\% dos casos, bem como a principal causa de uma elevada e importante morbidade e mortalidade. Por conseguinte, observa-se que diversos aspectos importantes relacionados à ruptura dos aneurismas intracranianos devem ser entendidos, para o tratamento mais adequado dos pacientes portadores desta patologia, com a finalidade de minimizar-se as suas morbidade e mortalidade ${ }^{3}$.

Em relação à hemorragia subaracnóidea (HSA) aneurismática, verifica-se que a mesma é mais comumente gerada em função da ruptura de aneurismas saculares, os quais se referem às lesões usualmente adquiridas, que resultam do estresse hemodinâmico prolongado e concomitante degeneração arterial nos pontos de saída dos principais ramos arteriais, bem como nas bifurcações das maiores artérias cerebrais que cursam nas cisternas e nos espaços subaracnóides ${ }^{4}$.

A ruptura de um aneurisma cerebral pode gerar hemorragia catastrófica num cérebro sadio, gerando com isto determinadas alterações graves no estado clínico do paciente, com presença de elevadas taxas de morbidade e mortalidade ${ }^{5}$. Estima-se que cerca de $12 \%$ dos pacientes morrem antes de receber o tratamento medicamentoso ${ }^{6}$. Nesse contexto, $40 \%$ dos pacientes hospitalizados morrem com um mês após o evento, e mais de um terço dos que sobrevivem vem a apresentar elevados déficits neurológicos ${ }^{7}$.

É importante que a haja uma coordenação que permitia a melhor eficiência da equipe multiprofissional para tratar o paciente com AVC hemorrágico, reduzindo o tempo de internação e a mortalidade hospitalar. O enfermeiro tem o papel fundamental no tratamento do paciente com HSA, deve-se incluir a obtenção de todas as informações necessárias, sua análise e síntese para a elaboração do plano de cuidado, se baseando no que cada paciente necessita quanto a terapêutica de cuidado proposta. Com isso, o presente artigo busca Identificar os elementos que caracterizam a HSA e propor medidas de atendimento às vítimas com suspeita de HSA na sala de Emergência.

\section{METODOS}

Trata-se de uma pesquisa bibliográfica que permite ao pesquisador ter contato direto com tudo que já foi descrito, dito ou filmado, sobre determinado assunto, o que não seria possível se ele fosse a campo efetuar sua própria pesquisa. Esse método não é mera repetição do material existente, uma vez que propicia novo 
exame sobre o tema, a partir de um novo enfoque ou nova abordagem, chegando, também, a novas conclusões.

A conduta metodológica adotada foi a da pesquisa integrativa, seguindo-se os passos: a elaboração da questão norteadora; busca ou amostragem na literatura; coleta de dados; análise crítica dos estudos incluídos; discussão dos resultados e apresentação dos resultados da pesquisa $^{8}$.

Foram priorizados, durante a pesquisa dos dados, os meios eletrônicos que representam atualmente a democratização do acesso às informações e possibilidade de atualização permanente. Foram realizadas buscas nas bases de dados da Biblioteca Virtual de Saúde (BVS), a Scientific Eletronic Library Online (SCIELO), MedLine, no Google Acadêmico, além das bibliotecas virtuais de instituições de ensino superior brasileiras.

Estabeleceu-se como critério inicial de seleção dos materiais: publicações em língua portuguesa, com texto completo, que abordassem em seu conteúdo a temática proposta, ou seja, pacientes com hemorragia subaracnóidea em unidades de alta complexidade.

\section{RESULTADOS}

\subsection{A Hemorragia Subaracnóidea.}

A denominada Hemorragia subaracnóidea (HSA) aneurismática é considerada como sendo um evento clínico grave que se caracteriza por ruptura e sangramento abrupto, ficando o sangue usualmente limitado ao espaço do líquido cefalorraquidiano (LCR), compreendido entre as membranas pia-máter e aracnoide ${ }^{9,10}$.

Observa-se que a incidência de HSA permaneceu estável durante as últimas três décadas, em um nível em torno de 6 casos por 100.000 pacientes/ano. Qualquer redução aparente é atribuível a uma proporção crescente de pacientes investigados com tomografia computadorizada (TC), por meio da qual são excluídas outras condições hemorrágicas ${ }^{11}$.

Em relação à taxa de mortalidade nos indivíduos com HSA, considera-se que a mesma chega a um patamar de 40\%, sendo esta responsável por $5 \%$ de todos os eventos de disfunção neurológica onde a manifestação inicial se caracteriza por quadro clínico de início súbito $^{12}$. Sobre a evolução dos pacientes com HSA, a mesma apresenta uma taxa expressiva de morbidade, a qual atinge 40 a 50\% dos pacientes que sobrevivem à crise inicial ${ }^{10,12}$. Além disso, existe a ocorrência frequente de sequelas nos pacientes sobreviventes, dos quais cerca de um terço acaba apresentando algum tipo de distúrbio, seja motor, cognitivo ou mesmo comportamental ${ }^{12,13}$.

A idade média de ocorrência da hemorragia subaracnóidea varia entre os diversos estudos pesquisados, mas normalmente compreendido na faixa etária de 50 a 52,6 anos. Pacientes $>70$ anos normalmente apresentam um quadro mais grave. Além disso, somente 2\% dos aneurismas se verificam durante a infância ${ }^{14,15}$. 
Com o aumento da idade, as possibilidades de um aneurisma romper ampliam-se, sendo que o pico de incidência ocorre por volta dos 60 anos de idade $^{16}$. Em um estudo identificado nesta pesquisa, envolvendo 76 pacientes com HSA, pode se verificar que a taxa de mortalidade nos indivíduos com mais de 60 anos girava em torno de $48 \%$, enquanto que em indivíduos mais jovens a taxa foi de $19 \%{ }^{17}$.

Deve-se ressaltar que na forma espontânea, os aneurismas correspondem a 75$80 \%$ das hemorragias, sendo que as malformações arteriovenosas representam 4-5\% desses eventos. Também observa-se a existência de outras situações menos frequentes, tais como vasculites que envolvem o sistema nervoso central (SNC), distúrbios de coagulação, tumores, dissecção de artéria cerebral e doença falciforme, entre outras, possivelmente por causas desconhecidas em 14$22 \%$ dos $\operatorname{casos}^{12,13}$.

É importante destacar que a ruptura de um aneurisma encefálico apresenta um elevado índice de mortalidade no primeiro episódio, e estima-se que aproximadamente 50\% evoluem para óbito antes do primeiro atendimento. Os sobreviventes apresentam um risco de ressangramento de 60\% nos três meses seguintes10,18. Ademais, considerase que a abordagem microcirúrgica é considerada como uma boa opção de tratamento. $\mathrm{Na}$ literatura, verifica-se que alguns estudos, demonstram que ocorreu uma tendência mundial para o tratamento endovascular, e este tratamento propiciou a condução terapêutica nos aneurismas não $\operatorname{rotos}^{19}$.
Observa-se que uma alteração que pode ser desencadeada com a ocorrência do extravasamento sanguíneo no espaço subaracnóideo se refere à disfunção olfatória, provavelmente pela proximidade anatômica do local da hemorragia com o sistema olfatório, estando sujeito a determinados fatores associados à HSA aneurismática, tais como dano mecânico direto nos tecidos, processos inflamatórios, aumento de pressão intracraniana e isquemia regional cortical ${ }^{19,20}$.

Ademais, em decorrência das suas características, o tratamento cirúrgico para HSA aneurismática também pode influenciar o olfato. Considera-se, nesse caso, que as alterações nessa função podem decorrer por meio do impacto da tração de tecidos cerebrais durante a exposição do sítio hemorrágico ${ }^{19}$.

Nota-se que os sintomas da HSA normalmente ocorrem de forma abrupta. Pequenas hemorragias podem apresentar-se assintomáticas, mas quanto maiores, maior a possibilidade de apresentar determinados sintomas, tais como dor de cabeça, vômitos e rebaixamento do nível de consciência e maior a taxa de morbimortalidade ${ }^{21}$.

\section{A HSA apresenta duas principais} origens:

a) Rompimento de aneurismas em torno de $85 \%$ dos casos em artérias da base do cérebro;

b) Sangramento de malformações vasculares na superfície cerebral ${ }^{22}$. 
No caso do sangramento ser resultante da ruptura de um aneurisma, o sangue se mistura ao Liquido Céfalo-Raquidiano (LCR), levando rapidamente a um aumento da Pressão Intracaniana (PIC); esse sangramento costuma durar pouco tempo, mas a possibilidade de um novo sangramento é elevada ${ }^{23}$.

Conforme mencionado, os sintomas ocorrem de forma abrupta, já que a PIC aumenta rapidamente pela mistura do sangue com o LCR, podendo culminar com déficits, tais como perda de memória. A cefaleia aparece comumente em todos os casos, apesentando forte intensidade e se espalha por toda a cabeça, podendo irradiar para a nuca ou até para as costas e membros inferiores ${ }^{18}$.

Nãoé comum a ocorrência de déficit focal, o que pode ocorrer quando de uma hemorragia intracerebral concomitante, caracterizando assim, a hemorragia meningocerebral. Em comparação à Hemorragia Intracerebral $(\mathrm{HI})$, dessa forma, a dor de cabeça no início do quadro, é mais comum referida cefaleia é relacionada à presença de vômito (comum em HSA), que é altamente incomum em AVCi. A presença de cefaleia de forte intensidade e início abrupto pode ser o único sinal de uma HSA menor, que precede uma HSA maior (cefaleia sentinela), por isso tem sempre de ser valorizada ${ }^{21}$.

\subsection{Os cuidados de Enfermagem.}

Busca-se que o reconhecimento das manifestações clínicas e o preciso diagnóstico da hemorragia subaracnóidea sejam estratégias fundamentais para modificar o impacto produzido por esta doença. O enfermeiro deve estar preparado para intervir nos cuidados básicos de uma unidade de terapia intensiva (UTI) ou Unidade de Emergência e deve apresentar preparo para cuidados avançados, mantendo-se atualizado com a literatura e acompanhando os avanços tecnológicos, focando no paciente e sua melhora.

A avaliação rigorosa dos pacientes suspeitos dessa hemorragia na unidade de emergência visa um atendimento resolutivo, tornando-se um desafio para a equipe equilibrar a velocidade e a competência com questões éticas e legais ${ }^{25}$. O enfermeiro da unidade de emergência, baseado num protocolo de atendimento, ou seja, sistematizando a assistência de Enfermagem, para esta hemorragia poderá contribuir de forma significativa para, além da identificação desta neuropatologia, o adequado tratamento, evitando complicações potenciais desta afecção, assim como um melhor prognóstico das vítimas quando possível.

A abordagem hospitalar precisa ser rápida e eficaz,

evitando complicações secundárias a HSA e postergações da alta hospitalar ${ }^{1,26}$.

A fase subaguda precisa ser bem conduzida, como uma maneira de diminuir os índices de sequelas, como o comprometimento motor e cognitivo decorrentes da aneurisma cerebral. Além disto, retrata em seu estudo os 
fatores de riscos que levam esses pacientes à serem hospitalizados decorrente ao rompimento da aneurisma, tendo uma maior porcentagem a Hipertensão Arterial (74\%), o Diabetes Mellitus $(25 \%)$ e o tabagismo (22\%). Além de descrever os grandes problemas de acordo com o tempo de internação hospitalar, a qual tem a média de 11 dias, quanto mais o paciente é dependente da terapia hospitalar, mais propício ele fica à complicações devido a dispositivos invasivos, tendo como solução a análise da necessidade dos dispositivos e diminuição do tempo hospitalar26.

O cuidado de enfermagem é o que pode fazer o diferencial na assistência prestada a essa clientela, pois ele se dá de forma completa e focado no cuidado pleno do indivíduo. Embora esteja em um conturbado ambiente de aparelhagens múltiplas e desconforto, ele consegue agir de forma ampla e diferenciada. O enfermeiro por esta mais perto do paciente e ter uma formação mais voltada para humanização, consegue diminuir a impessoalidade, falta de privacidade, isolamento social, dentre outros problemas que ambientes de alta tecnologia possuem. Isso sem dúvida, fará a diferença na prestação do cuidado ao paciente vítima de HSA.

\section{CONSIDERAÇÕES FINAIS}

A HSA é uma alteração neurológica que muitas vezes representa uma emergência neurocirúrgica, normalmente o sangue fica restrito ao espaço do LCR, entre as membranas pia-máter e aracnoide. Sendo que a ruptura de aneurismas cerebrais é a causa mais comum de hemorragia não traumática subaracnóidea.

O índice de mortalidade é elevado neste grupo de pacientes, e em torno de 50\% deles vão a óbito antes mesmo de terem acesso a um atendimento hospitalar. Além disso, em função do aumento da idade, as possibilidades de um aneurisma romper aumentam, sendo que o pico de incidência ocorre por em torno dos 60 anos de idade.

Destaca-se que os fatores de risco estabelecidos para mortalidade incluem necessariamente: grau clínico ruim à admissão no serviço de saúde, idade avançada, ressangramento aneurismático, aneurisma de grande tamanho e isquemia cerebral por vasoespasmo.

Os profissionais de enfermagem são primordiais para um bom resultado da das condutas terapêuticas priorizadas para o paciente com HSA. É de fundamental importância a realização de capacitações, agregando conhecimentos para implementar os planos de cuidados, gerando um guia de ações focado nos planejamentos e medidas, com enfoque na humanização e principalmente a contínua sensibilização dos profissionais para a importância do diagnóstico precoce e o correto manejo da HSA.

É imperioso que as evidências científicas sejam o norte de atuação das equipes, priorizando sempre o paciente e a qualidade da assistência, focando principalmente na segurança do paciente, que é o ponto crucial do cuidado do 
enfermeiro ao paciente com HSA, nos cenários

de alta complexidade.

\section{REFERÊNCIAS}

1. LOUREIRO AB; VIVAS MC; CACHO RO; CACHO EWA; BORGES G. Evolução Funcional de Pacientes com Hemorragia Subaracnóide Aneurismática não Traumática. R Bras Ci Saúde 19(2):123-128, 2015.

2. TURCATO C, PEREIRA SW, GHIZONI MF. Hemorragia subaracnóide. Arquivos Catarinenses de Medicina, 2006; 35(2): 78-84.

3. PEREDA ALC; FALERO RAP; MACHIN PLV. Hemorragia Subaracnóidea. (2012). Disponível em: <http:// neuroc99.sld.cu/text/HSAMonografia.htm>. Acessado em Abr. 2021.

4. STEHNBENS WE. Etiology of intracranial berry aneurysms. J Neurosurg. 1989; 70(6): 823-831.

5. BORGES G. Tratamento cirúrgico e fatores preditivos da hemorragia subaracnóide. [Tese - Livre-Docência]. Campinas (SP): Universidade Estadual de Campinas; 2003.

6. SCHIEVINK WI. Intracranial aneurysms. N Engl J Med. 1997; 336(1): 28-40. DOI: 10.1056/ NEJM199701023360106.

7. IGAWA T, TOKUDA Y, OHBAYASHI N, TAKYA M, MORI TAKE K. Study of aneurysmal subarachnoid hemorrhage in Izumo city, Japan. Stroke. 1995; 26(5): 761-766.

8. MARCONI, M. A.; LAKATOS, E. M. Fundamentos de metodologia científica. 7 ed. São Paulo/SP: Atlas, 2010.

9. PULSINELLI WA. Doenças vasculares cerebrais princípios. In: GOLDMAN L, BENNETT JC. CECIL: tratado de medicina interna. 24a . Ed. Elsevier, 2014.

10. PEREIRA BJA, NAKASONE FJ, OLIVEIRA, JG. Cerebral vasospasm after aneurysmal subarachnoid hemorrhage: an uptodate review. Jornal Brasileiro de Neurocirurgia 2013. Maio 23, 24 (3): 224 - 241, 2013.

11. VAN GIJN J, RINKEL GJE. Subarachnoid haemorrhage: diagnosis, causes and management. Brain 2001, Feb; 124(2):249-78.

12. BURGOS ER, DIAZ RC. Hemorragia subaracnóidea espontanea: diagnostico y tratamiento. Univ. med. 2002; 43(4):260-5.

13. MOCCO J, KOMOTAR RJ, LAVINE SD, MEYERS PM, CONNOLLY S, SOLOMON R. The natural history of unruptured intracranial aneurysms. Neurosurg Focus. 2004;17(5): E3.

14. MALINOVA V; SCHATLO B; VOIT M, SUNTHEIM P, ROHDE V, MIELKE D. Identification of specific age groups with a high risk for developing cerebral vasospasm after aneurysmal subarachnoid hemorrhage. Neurosurgery Rev 2016 Jul; 39(3): 429 436.

15. GREENBERG MS. Manual de neurocirurgia. $7^{\mathrm{a}}$. Ed. Porto Alegre: Artmed; 2012. p. 728-73.

16. CLINCHOT DM, KAPLAN P, MURRAY DM, PEASE WS. Cerebral aneurysms and arteriovenous malformations: implications for rehabilitation. Arch Phys Med Rehabil. 1994;75(12):1342-51.

17. KLOSTER R. Subarachnoid hemorrhage in Vestfold county. Occurrence and prognosis. Tidsskr Nor Laegeforen. 1997 May 20; 17(13):1879-82.

18. OLIVEIRA BRL. Situações que MIMETIZAM AVC: uma revisão de literatura. Monografia de Conclusão do Componente Curricular MED-B60, e como pré-requisito obrigatório e parcial à conclusão do curso médico da Faculdade de Medicina da Bahia da Universidade Federal da Bahia, apresentada ao Colegiado do Curso de Graduação em Medicina. Salvador, BA, 2013.

19. GRIZ MFL. Relação entre déficit da olfação e hemorragia subaracnóidea aneurismática antes e após tratamento. Tese de doutorado. Recife: Universidade Federal de Pernambuco; 2014.

20. CROWLEY RW, MEDEL R, KASSELL NF, DUMONT AS. New insights into the causes and therapy of cerebral vasospasm following subaracnoided hemorrhage. Drug Discov Today. 2008;13(5-6):254-60.

21. CAPLAN, LR., et al. Etiology and classification of stroke. Uptodate, dez. 2012.

22. COELHO LG, COSTA JM, SILVA EI. Hemorragia subaracnóidea espontânea não aneurismática. Rev Bras Ter Intensiva. 2016;28(2):141-146

23. YEW, KS; CHENG, E. Acute Stroke Diagnosis. American Family Physician, n 1: 33-40, Jul. 2009.

24. SOUZA, MP. Enfermagem na Assistência ao paciente com Acidente Vascular

Cerebral em Ambiente Intra-Hospitalar. Rev. enferm. UERJ, RJ 2012.

25. Torreão LA. Aspectos éticos na emergência. AMB rev. Assoc. Med. Bras. [periódico na Internet]. 2003[Acesso em 2021 Jun 04]; 49(1). Disponível em: http://www. scielo.br/pdf/ramb/v49n1/15364.pdf.

26. MELO, LS; ET AL. Acidente Vascular Cerebral: Achados Clínicos e Principais

Complicações. Rev. Aten. Saúde, São Caetano do Sul, v. 14 , n. 48, p. 48-53, abr./jun., 2016.

OBSERVAÇÃO: Os autores declaram não existir conflitos de interesse de qualquer natureza. 